\title{
Anabases
}

ANABASES Traditions et réceptions de l'Antiquité

16 | 2012

Varia

\section{Larissa BONFANTE (éd.), The Barbarians of Ancient Europe. Realities and Interactions}

Claire Joncheray

\section{OpenEdition}

Journals

Édition électronique

URL : http://journals.openedition.org/anabases/4000

DOI : 10.4000/anabases.4000

ISSN : 2256-9421

Éditeur

E.R.A.S.M.E.

\section{Édition imprimée}

Date de publication : 1 octobre 2012

Pagination : 298-300

ISSN : 1774-4296

\section{Référence électronique}

Claire Joncheray, "Larissa Bonfante (éd.), The Barbarians of Ancient Europe. Realities and Interactions », Anabases [En ligne], 16 | 2012, mis en ligne le 01 octobre 2012, consulté le 22 septembre 2020. URL: http://journals.openedition.org/anabases/4000 ; DOI : https://doi.org/10.4000/anabases.4000

Ce document a été généré automatiquement le 22 septembre 2020.

(c) Anabases 


\title{
Larissa BONFANTE (éd.), The Barbarians of Ancient Europe. Realities and Interactions
}

\author{
Claire Joncheray
}

\section{RÉFÉRENCE}

Larissa BONFANTE (éd.), The Barbarians of Ancient Europe. Realities and Interactions, Cambridge et New York, Cambridge University Press, 2011, 424 p.

60 livres / ISBN 978-0-521-19404-4.

1 Les populations du bassin septentrional de la Méditerranée qui ne possèdent pas de littérature historique propre et sont cataloguées par leurs us et coutumes sous l'étiquette de "barbares" dans la littérature gréco-latine font l'objet de ce recueil d'articles. Leur étude se présente non de manière exhaustive, mais dans la perspective d'une réflexion articulant les textes et l'archéologie. Les contributeurs se proposent de concentrer l'attention sur les Scythes, les Thraces, les Étrusques, le monde alpin, les Germains, les Celtes des îles anglo-saxonnes, les Romains et les Goths.

2 La publication, en langue anglaise, des conférences prononcées à l'université de Richmond en 2003 comprend une introduction conceptuelle par l'éditeur, Larissa Bonfante, dix contributions, une conclusion de Barry Cunliffe, enfin une note sur le tableau de Delacroix intitulé Ovide chez les Scythes par Ann E. Farkas, qui centre son étude sur le sentiment d'isolement du poète par rapport à une doxa.

L'excellente introduction permet un bon recadrage de l'ensemble des critères qui participent de la qualification d'un barbare dans sa confrontation avec les pratiques grecques notamment (comme les habits, la place des femmes ou le rituel du sacrifice humain). En complément, l'article de Paul Keyser (Greek geography of western barbarians) présente chronologiquement les auteurs grecs dont les textes sont à l'origine de ces critères et d'une typologie sur les types de barbares. Toutefois la conclusion, qui prend 
acte de la faiblesse des sources historiques et de la nécessité d'étudier les sociétés dans leurs interactions, ne tient pas compte du débat sur le concept de méditerranéeisation (P. Horden et N. Purcell, The corrupting sea, Oxford, Blackwell, 2000) et, par conséquent, du fait que la Méditerranée n'est pas forcément le centre mais plutôt une périphérie pour chacune des sociétés dites "barbares ». Les cartes générales de l'Europe ne sont d'ailleurs pas utilisables à cause de la mauvaise localisation de certains sites, comme celui de Vix.

Deux articles se concentrent sur les Scythes: Askold Ivantchik (The funeral of Scythian kings: the historical reality and the description of Herodotus) relit le texte d'Hérodote comme une source historique, en montrant que l'historien grec utilise des sources anciennes qui remontent au $\mathrm{VIII}^{\mathrm{e}}$ siècle, pour la description du rituel funéraire d'ensevelissement royal, alors que d'autres thématiques, comme la localisation des tombes, correspondent bien aux pratiques scythes qui lui sont contemporaines. Renate Rolle (Scythians: between mobility, tomb architecture and early urban structures) apporte une nouvelle donnée archéologique essentielle pour l'étude de l'urbanisme protohistorique avec le site de Gorodische, une massive forteresse d'Ukraine, disposant à l'intérieur de deux autres espaces entourés eux-mêmes de remparts. L'hypothèse d'une vocation de résidence royale, en plus d'une vocation commerciale du site, est séduisante parce qu'elle permet de maintenir la vision d'un nomadisme royal, ce site offrant en même temps un vaste espace protégé par des remparts pour recevoir les caravanes royales lors de leurs déplacements. Les Thraces sont étudiés par Ivan Marazov (Philomele's tongue : pictorial language of myth in ancient Thrace) afin de montrer combien l'art thrace est soumis aux besoins idéologiques des rois en se fondant sur des images de bravoure, malgré un répertoire assez disparate et parfois inspiré des images grecques. L'appendice sur les nouvelles découvertes en Bulgarie est très utile.

5 Le panorama des populations européennes se prolonge d'Est en Ouest par le monde celte. L'identité de ce dernier correspond à des fabrications littéraires principalement romaines qui n'ont pas d'écho dans les vestiges archéologiques. Le nom des Celtes, pour les populations de l'Irlande et de la Grande Bretagne d'après Barry Cunliffe (In the fabulous Celtic twilight) ne permet par de rendre compte des millénaires d'interactions culturelles et des grandes différences spatio-chronologiques. Les Germains, localisés de Mayence jusqu'au nord du Danemark, subissent, d'après Peter S. Wells (The ancient Germans), un sort semblable, dans la mesure où les traces archéologiques ne permettent pas de déterminer les différents faciès culturels des tribus et que le mot «Germain » correspond davantage à une fabrication romaine qu'à une conscience ethnique existante. Enfin, les Goths, vus par les Romains comme les descendants des Germains, semblent se comporter comme des chrétiens: Walter Stevenson (The identity of late barbarians: Goths and wine) utilise l'exemple de la fabrication du vin pour montrer comment des populations disparates formant le peuple goth souhaitent une indépendance culturelle et économique.

6 Pour le monde du nord de l'Adriatique, les sociétés semblent davantage se reconnaître dans une culture qui leur est propre, même si elles s'expriment par des moyens iconographiques et alphabétiques importés. Les Étrusques, nourris de culture grecque, filtrent les données méditerranéennes et, par leurs liens commerciaux anciens et continus jusqu'au $\mathrm{IV}^{\mathrm{e}}$ siècle av. J.-C., diffusent des motifs iconographiques repris en Europe centrale et dans le monde des situles (Larissa Bonfante, The Etruscans : mediators between northern barbarians and classical civilization, et Otto-Herman Frey, The world of 
situla art). À partir de l'exemple des têtes coupées, Nancy Thomson de Grummond (A barbarian myth? The case of the talking head) montre de manière convaincante comment un même motif iconographique revêt des réalités mythologiques et sociales différentes entre le monde grec, à travers le mythe d'Orphée, le monde étrusque, à travers des figures prophétiques et les mondes celtes, à travers le prestige guerrier. Pour les Celtes du Midi, il est possible de nourrir cette réflexion à partir des données du catalogue édité par R. Roure et L. Pernet, Des rites et des hommes (Paris, Errance, 2011). Enfin le dernier peuple étudié, les Romains, ne correspond pas à la conception grecque du barbare (John Marincola, Romans and/as barbarians): le changement sémantique de cette notion et l'évolution des critères qui lui sont attribués apparaissent ainsi de manière flagrante.

7 À travers une série d'exemples, la plupart des peuples de l'Europe dans l'Antiquité acquiert une autonomie culturelle et sociale dans les études scientifiques actuelles. Il semble donc que la connaissance des mœurs méditerranéennes passe principalement par des images mythologiques avant qu'un contact direct et violent, notamment avec Rome, n'oblige les peuples à se définir eux-mêmes et à imposer de nouveaux critères de distinction culturelle et sociale.

\section{AUTEURS}

\section{CLAIRE JONCHERAY}

Université d'Aix-Marseille

joncheray@mmsh.univ-aix.fr 